FORMATION Formation emploi

Revue française de sciences sociales

120 | Octobre-Décembre 2012

Parcours de formation : la recomposition des

ségrégations

\title{
Vingt ans de politiques de rééquilibrage en Nouvelle-Calédonie : Démocratisation de l'école mais persistance des inégalités ethniques
}

Twenty years of "Closign the gap" policies in New Caledonia: Democratization of School and ethnic inequalities

20 Jahre Ausgleichspolitik in Neukaledonien:

Demokratisierung der Schule, aber Fortbestehen der ethnischen Ungleichheit

Veinte años de políticas de reequilibración en Nouvelle-Calédonie:

democratización de la escuela pero persistencia de las desigualdades étnicas

Laure Hadj, Gaël Lagadec, Gérard Lavigne et Catherine Ris

\section{(2) OpenEdition \\ Journals}

Édition électronique

URL : http://journals.openedition.org/formationemploi/3855

DOI : 10.4000/formationemploi.3855

ISSN : 2107-0946

Éditeur

La Documentation française

Édition imprimée

Date de publication : 31 octobre 2012

Pagination : 101-125

ISSN : 0759-6340

Référence électronique

Laure Hadj, Gaël Lagadec, Gérard Lavigne et Catherine Ris, « Vingt ans de politiques de rééquilibrage en Nouvelle-Calédonie : Démocratisation de l'école mais persistance des inégalités ethniques »,

Formation emploi [En ligne], 120 | Octobre-Décembre 2012, mis en ligne le 15 février 2013, consulté le 30 octobre 2020. URL : http://journals.openedition.org/formationemploi/3855 ; DOI : https://doi.org/ 10.4000/formationemploi.3855 


\title{
Vingt ans de politiques de rééquilibrage en Nouvelle-Calédonie
}

\section{Démocratisation de l'école mais persistance des inégalités ethniques}

\begin{abstract}
LAURE HaDJ
Doctorante en socio-démographie au CEPED (Centre Population et Développement) à I'université Paris 5 Sorbonne. Elle effectue sa thèse en partenariat avec I'Institut de la statistique et des études économiques (ISEE) de Nouvelle-Calédonie où elle est chargée d'études

GAËL LAGADEC Maître de conférences en sciences économiques à l'université de la Nouvelle-Calédonie. Il poursuit ses recherches au sein du Larje (Laboratoire de recherches juridiques et économiques) de l'université de la Nouvelle-Calédonie

GÉRARD LAVIGNE Doctorant ethno-mathématiques au CNEP (Centre des nouvelles études sur le Pacifique) à I'université de la Nouvelle-Calédonie

CATHERINE RIS Maîtresse de conférences en sciences économiques à l'université de la Nouvelle-Calédonie. Elle poursuit ses recherches au sein du Larje (Laboratoire de recherches juridiques et économiques) à l'université de la Nouvelle-Calédonie
\end{abstract}

Résumé

Vingt ans de politiques de rééquilibrage en Nouvelle-Calédonie. Démocratisation de l'école mais persistance des inégalités ethniques

Selon les quatre derniers recensements de la population (1989, 1996, 2004, 2009), le niveau de qualification de la population calédonienne a fortement progressé. Cependant, les inégalités ethniques devant l'école persistent et s'accentuent aux extrêmes : les nondiplômés et les diplômés de l'enseignement professionnel sont très majoritairement Kanaks, les diplômés du supérieur très majoritairement non Kanak. Ces inégalités renvoient aux écarts dans la maîtrise des fondamentaux observés dès le CE2. Elles se sont cependant réduites. Ainsi cette «massification " de l'accès aux diplômes s'accompagnerait d'une "démocratisation qualitative " de l'école, dont nous discutons toutefois la portée. 
Mots clés : Recensement, niveau de formation, discrimination raciale, inégalités, politique de l'éducation, Nouvelle Calédonie

Abstract

Twenty years of "Closign the gap" policies in New Caledonia: Democratization of School and ethnic inequalities

Using data from the last four censuses (1989, 1996, 2004, 2009), we find that the skill level of the New Caledonian population rose sharply for all communities. However, ethnic inequalities remain important and increase with the level of the diploma, e.g. most university graduates are non-Kanak. This implies that inequalities, which already appear in primary school, may widen over the lifecycle, a finding that has implications for policies aiming to close the gap between indigenous and non-indigenous people. However inequalities decreased between 1989 and 2009. This suggests that the "massification" of access to diplomas may be accompanied by a "qualitative democratization" of school in New Caledonia.

Keywords: Census, training level, racial discrimination, inequalities, education policy, New Caledonia

Journal of Economic Literature: I 28, J 15

Traduction : Auteurs

Petite économie insulaire du Pacifique Sud, la Nouvelle-Calédonie se distingue dans l'ensemble de l'outre-mer français, et plus encore en Océanie, par un niveau de vie élevé et un indice de développement humain qui la place en 34ème position mondiale. Le PIB (produit intérieur brut) par habitant est aujourd'hui comparable à celui de la grande majorité des régions françaises et est supérieur à celui de la Nouvelle-Zélande. Les dix dernières années ont été marquées par un progrès économique et social important, notamment grâce aux investissements dans l'exploitation du nickel, ressource naturelle constituant la principale richesse de l'île. La société calédonienne est cependant marquée par de fortes inégalités, qu'elles soient monétaires, sociales, géographiques ou ethniques. Ces inégalités s'accompagnent d'inégalités persistantes dans le système éducatif et sur le marché du travail. Depuis les signatures des Accords de Matignon (1988) et de Nouméa (1998), la Nouvelle-Calédonie s'est engagée sur la voie du "rééquilibrage » en faveur de la communauté Kanak, peuple autochtone, longtemps discriminé.

Des efforts ont ainsi été réalisés dans tous les domaines économiques et sociaux pour permettre aux deux provinces, Nord et Iles, majoritairement kanak, de " rattraper " la province Sud, majoritairement non kanak. Les politiques de quotas ou encore de discrimination positive visent également à diminuer les ségrégations dans le système éducatif ou sur le marché du travail. 
C'est au regard de ces vingt années de politiques de réduction des inégalités que sera analysée l'évolution de l'accès aux diplômes en Nouvelle-Calédonie, en attachant une attention particulière à l'évolution des inégalités ethniques.

Le recours aux statistiques ethniques n'est pas jugé contraire au principe d'égalité pour de nombreux États occidentaux, tels que la Grande-Bretagne, les États du Nord de l'Europe, du Nord de l'Amérique, qui utilisent largement, et depuis plus d'un siècle pour certains, cet outil pour mettre en œuvre des politiques publiques de réduction des inégalités à l'égard des minorités. Il en va différemment en France, où la conception républicaine du principe d'égalité constitue le principal obstacle au recours aux statistiques ethniques ${ }^{1}$. Cependant, « l'intérêt public » motive une dérogation à la loi informatique et libertés de 1978 qui énonce une interdiction de principe sur le traitement statistique des données sensibles (David, 2011).

C'est le cas en Nouvelle-Calédonie, où les neuf recensements effectués sur le territoire depuis la seconde guerre mondiale (sauf celui de 2004) ont tous identifié la communauté d'appartenance. La nature des informations contenues dans les recensements de la population calédonienne permet donc un traitement tout à fait original dans l'espace républicain français. En effet, si dans les pays anglo-saxons, les études mesurant l'évolution des inégalités ethniques sont nombreuses, en France, du fait de la limitation de l'utilisation des données ethniques, cette dimension est presque toujours absente des études.

La situation de la Nouvelle-Calédonie se distingue de celle de la France et de la plupart des pays occidentaux en ce que les ethnies discriminées ne sont pas celles issues de l'immigration. C'est bien du contraire qu'il s'agit : la Nouvelle-Calédonie a été une terre d'immigration (colonisation de peuplement) mais les inégalités observées existent à l'avantage des immigrants et au détriment du peuple autochtone. C'est en cela qu'elle se rapproche de ses voisins anglo-saxons de la région (Australie et Nouvelle-Zélande). Cependant, le groupe discriminé ici n’est pas un "groupe minoritaire »; les Kanaks représentent $40 \%$ de la population calédonienne en 2009.

Ces données chiffrées permettent ici précisément d'attester la réalité des plans d'action en matière de rééquilibrage entre les communautés; on s’attachera à mesurer l'évolution des inégalités devant l'école, mesurées par l'accès au diplôme. L'égalité des chances devant l'école est cependant une ambition assez récente en Nouvelle-Calédonie ; en effet, l'école publique läque n’a autorisé les Kanaks à se présenter au baccalauréat que vers le milieu du siècle dernier (premier bachelier kanak en 1962).

En Nouvelle-Calédonie, comme en Europe, on assiste à un allongement général des études. Il est important de vérifier que cette «massification de l'école » s'accompagne d'une réduction du lien entre origine et destinée scolaire, ce qui correspond à une « démocratisation qualitative de l'école ». Cette question ne concerne pas uniquement l'égalité des chances à l'école puisque,

1. Pour le COMEDD (Comité pour la mesure et l'évaluation de la diversité et des discriminations) (2010), ce principe d'égalité proclamé dans l'absolu conduit à nier la complexité des situations et à perdre toute prise sur la réalité. 
au-delà, les inégalités dans l'acquisition de compétences ont un impact sur l'insertion sur le marché du travail et en particulier la constitution des élites de la société (Thélot et Vallet, 2000). Réussir à l'école est devenu une condition quasi nécessaire à l'accès à l'emploi. Les inégalités devant l'école pèsent donc également sur l'insertion sociale. Ces inégalités peuvent concerner la mobilité sociale, comme cela a largement été étudié en France, ou la discrimination à l'égard de groupes minoritaires (Beauchemin, Hamel, Lesné et Simon, 2010), notamment ethniques, comme cela est particulièrement mis en avant dans les pays anglo-saxons (voir Fryer, 2010, pour l'exemple des USA).

L'objet de cet article est de proposer une mesure de l'évolution, sur les deux dernières décennies, de l'accès des différentes communautés aux diplômes. Aucun travail descriptif systématique n’a jamais été mené sur ce point en Nouvelle-Calédonie.

Les principaux résultats sont résumés ci-dessous :

- Dans l'ensemble, sur les vingt dernières années, on assiste à une forte progression du niveau d'éducation dans toute la population calédonienne ;

- Cependant, à toutes les dates des recensements, les Kanaks sont moins diplômés que les non-Kanaks ;

- Le calcul des rapports de chances relatives montre que l'écart entre Kanaks et non-Kanaks augmente avec le niveau du diplôme : écart de 1 à 2,8 pour n'importe quel diplôme, de 1 à 4,8 pour le baccalauréat toutes mentions, de 1 à 6,1 pour le baccalauréat général et de 1 à 9,4 pour les diplômes de l'enseignement supérieur ${ }^{2}$;

- Une exception notable : il n'y a que pour les baccalauréats technologiques et professionnels que l'écart entre les communautés est très faible ;

- Pour tous les diplômes, l'écart entre les communautés se réduit entre 1989 et 2009.

L'appréciation globale de réduction des inégalités est donc à nuancer lorsque l'on s'intéresse aux détails des types de diplômes obtenus (notamment les séries de baccalauréat). On constate également que les disparités apparaissent dès l'école primaire (écarts dans la maîtrise des fondamentaux dès le CE2), s'accentuent en Gème et conduisent à une orientation en seconde professionnelle. Il apparaît donc que l'école calédonienne, en calquant l'école française, ne contribue pas pleinement à l'objectif d'égalité des chances devant l'école.

Dans une première partie, nous présentons le développement du système éducatif calédonien. La deuxième partie est consacrée à la présentation de la littérature sur la mesure des inégalités ethniques. Dans une troisième partie, nous analysons l'évolution des inégalités ethniques dans le cadre des politiques publiques de rééquilibrage. Enfin, dans une quatrième partie, nous revenons sur le parcours scolaire des individus pour montrer que les inégalités dans la réussite scolaire apparaissent très tôt et se creusent au fur et à mesure de l'allongement des études.

2. En 2009, un non-Kanak a 9,4 fois plus de chances qu'un Kanak d'obtenir un diplôme de l'enseignement supérieur. 


\section{L'école calédonienne dans un environnement économique et culturel riche}

\subsection{Une collectivité sui generis d'outre-mer riche mais marquée par les inégalités}

\section{Carte 1}

Les Pays et territoires du Pacifique

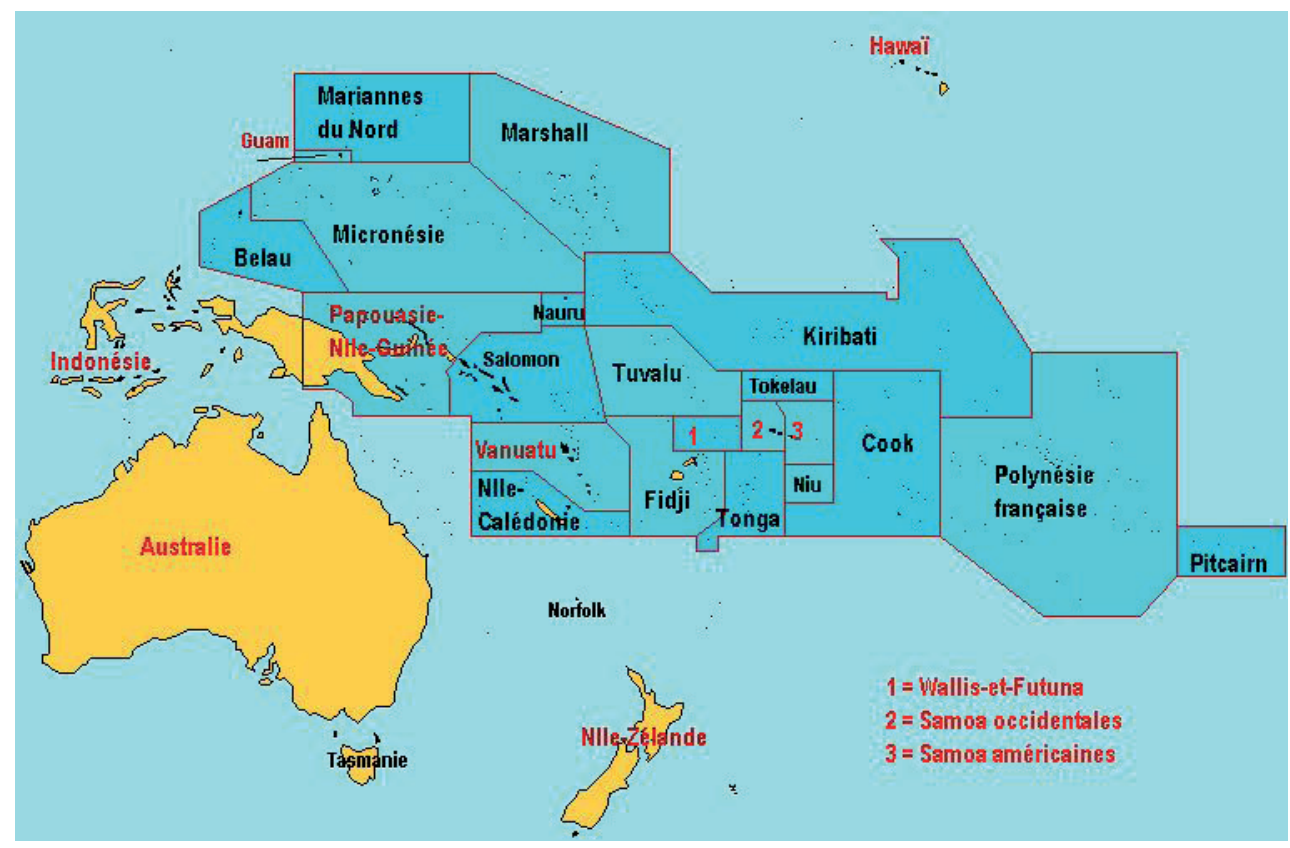

Source : Commission du Pacifique Sud (CPS, Nouméa).

N. B : « La Nouvelle-Calédonie est constituée de trois provinces, les provinces Nord et Sud, et les îles Loyautés. En 2009, elle comptait 245580 habitants. Parmi les jeunes, des déséquilibres communautaires subsistent ; ainsi, 54,1\% des Européens possèdent le baccalauréat, contre 12,5\% des Kanaks et 14,2 \% des Wallisiens et Futuniens (source Insee : Insee Première n ${ }^{\circ} 1338$ - février 2011.

Depuis l'Accord de Nouméa (1998) qui encadre le statut et l'avenir institutionnel de la Nouvelle-Calédonie, la croissance calédonienne est soutenue et régulière : + 3,7\% en moyenne annuelle entre 1998 et 2008. La société calédonienne est cependant marquée par de fortes inégalités ${ }^{3}$, qu'elles soient d'origines sociales, monétaires, géographiques ou ethniques. Les provinces Nord et des Iles Loyauté, qui comptent une population majori-

3. Les inégalités de niveau de vie sont élevées : les plus modestes ont un niveau de vie 9,7 fois plus faible que celui des plus aisés (Hadj, 2010). 
tairement kanak, sont les moins riches et bénéficient d'infrastructures moins développées ; le revenu médian en province Sud est deux fois supérieur à celui de la province Nord et 2,5 fois supérieur à celui des îles Loyauté.

Il n'existe pas aujourd'hui, en Nouvelle-Calédonie, de source statistique permettant de connaître à la fois l'origine sociale et l'origine ethnique des individus. Nous utilisons ici les recensements de la population (1989, 1996, 2004 et 2009) ; ils permettent d'utiliser une variable d'appartenance communautaire mais ne rendent pas possible l'évaluation de la mobilité sociale en prenant en compte l'origine sociale et culturelle de l'individu. Tout en sachant que ces deux sources d'inégalités (sociales et ethniques) se renforcent mutuellement $^{4}$, on se focalise ici sur les inégalités ethniques, en distinguant Kanaks et non Kanaks. Le recensement de 2009 dénombre à peine un millier de cadres Kanaks. Seulement $3 \%$ des actifs kanaks sont cadres contre $13 \%$ dans le reste de la population. De même, les Kanak sont en proportion deux fois moins nombreux que les autres communautés à exercer une profession intermédiaire (Broustet et Rivoilan, 2012). Le groupe «non Kanak » présente une grande hétérogénéité (de par les différentes communautés qu'il rassemble 5 ) ; cependant, le choix de cette distinction communautaire se justifie d'une part pour rendre nos résultats comparables à ceux obtenus en Australie et Nouvelle-Zélande (où l'hétérogénéité au sein des groupes des non-autochtones est encore plus élevée $\left.e^{6}\right)$ et d'autre part, pour mesurer l'efficacité des politiques de rééquilibrage en faveur de la communauté Kanak dans l'esprit des Accords de Matignon (1988) et de Nouméa (1998).

Depuis les signatures de ces Accords, des mesures de politiques de réduction des inégalités ont été mises en place en faveur du peuple Kanak.

Le rééquilibrage économique interprovincial est mis en œuvre de diverses manières, dont les plus significatives sont les clés de répartition budgétaires. Les dotations aux provinces sont déséquilibrées dès lors qu'on raisonne en dotations par tête?. C'est l'objet du rééquilibrage. Cependant, alors que les clés du rééquilibrage sont, par définition, avantageuses pour le Nord et les Iles Loyauté, elles n'apparaissent pas suffisantes ; l'avance économique de la province Sud est considérable.

4. Le taux d'emploi en 2009 atteint $71 \%$ pour les Européens contre $45 \%$ pour les Kanaks. Seulement $3 \%$ des actifs kanaks sont cadres contre $13 \%$ dans le reste de la population (Broustet et Rivoilan, 2012).

5. Répartition de la population calédonienne par communauté d'appartenance selon le recensement de la population de 2009 : Kanak : $40 \%$, Européenne : $29 \%$, Wallisienne et Futurienne : $9 \%$, métis : $8 \%$, autres communautés : $7 \%$, Calédonienne : $5 \%$ déclarent appartenir à la communauté "calédonienne", refusant ainsi de choisir parmi les communautés proposées (ISEE, Synthèse Recensement de la population 2009 : http://www.isee.nc/population/telecharpdf/4\%20page-rpnc09.pdf).

6. En Australie, les Aborigènes et les autochtones du détroit de Torres représentent 2,3\% des 22 millions d'habitants. En Nouvelle-Zélande, les Maoris représentent $15 \%$ des 4,2 millions d'habitants.

7. Les dotations sont de $50 \%$ pour le Sud, $32 \%$ pour le Nord et $18 \%$ pour les Iles Loyauté alors que la répartition de la population est de $75 \%$ pour le Sud, $18 \%$ pour le Nord et $7 \%$ pour les Iles Loyauté. 
Pour poursuivre le rééquilibrage et l'accession des Kanaks aux responsabilités dans tous les secteurs d'activité, c'est dans le domaine de l'emploi et de la formation que les politiques de rééquilibrage sont les plus visibles. Des structures et des programmes de formation ont ainsi été mis en place à l'exemple du programme " 400 cadres ». Cette formation est destinée aux jeunes adultes qui ont achevé leurs études secondaires. L'objectif est de former, sur 10 ans, des cadres principalement Kanak, dans la plupart des secteurs d'activité. En 1998, la formation est renouvelée et renommée "Cadre avenir ».

Parallèlement à cela, afin de répondre à l'étroitesse du marché du travail calédonien, et dans le contexte de l'émancipation voulue par l'Accord de Nouméa, une politique de protection de l'emploi local a fait l'objet d'une loi de Pays (28 juillet 2010). Cette loi donne priorité à l'emploi aux citoyens calédoniens (c'est-à-dire les personnes nées en Calédonie ou s'y étant installées avant le référendum de 1998 sur l'Accord de Nouméa). Selon les difficultés de recrutement pour les différents types d'emplois, la loi prévoit des durées de résidence pour y avoir accès ; ces durées peuvent aller jusqu'à dix ans pour les emplois ne présentant aucune difficulté de recrutement.

Cependant, là non plus, les politiques en matière de formation et d'emploi ne parviennent pas à lisser les inégalités, notamment entre provinces, comme nous allons le voir.

\subsection{Le développement du système éducatif calédonien : une démocratisation quantitative}

L'école publique laïque, en Nouvelle-Calédonie, est officiellement instituée par l'arrêté du Gouverneur Guillain du 15 octobre 1863 (Guillain, 1863), «vu la nécessité de constituer, dans la colonie, un enseignement en rapport avec les besoins les plus immédiats " qui sont définis tels que : $: 1^{\circ}$ ) de pourvoir à l'instruction des enfants de colons; $2^{\circ}$ ) de faciliter nos relations avec les indigènes et l'accomplissement de notre mission civilisatrice à leur égard, ce qui exige avant tout quils nous comprennent. " La priorité pour l'ouverture de l'école publique, clairement exprimée, réside donc dans l'instruction des enfants de colons. Le dernier alinéa de l'article 2 précise que "l'étude des idiomes calédoniens est formellement interdite dans toutes les écoles ». Interdiction qui sera reprise dans les arrêtés successifs organisant l'école calédonienne et qui va perdurer jusqu'en 1984. Les écoles missionnaires, installées depuis 1841, distribuaient un enseignement, essentiellement religieux, en langue kanak. Ces écoles furent mises en demeure de se conformer à cette interdiction, sous peine de fermeture. L'école "des jeunes indigènes» avait été ouverte provisoirement en 1862 et organisée définitivement en 1863. Les priorités y étaient plus explicites quant à l'enseignement à dispenser aux jeunes kanak : il devait essentiellement consister à " approprier les jeunes Kanaks aux industries manuelles les plus utiles dans une société naissante".

La distinction entre ces écoles est d'importance : l'une d'enseignement général pour les enfants européens et l'autre d'enseignement professionnel pour certains enfants kanak. Ceci va marquer toute la fonction scolaire de la colonie. Ce fonctionnement séparé de 
l'école restera en vigueur jusqu'en 1946, avec la fin du code de l'indigénat, institué en 1887. Ce n'est qu'au milieu du XXème siècle, il n'y a donc pas si longtemps, que les Kanaks pourront accéder à l'école publique laïque comme les Européens.

Les enjeux et objectifs de l'école calédonienne sont clairement énoncés par les institutions (DENC, 2008) :

- "Adapter le système éducatif calédonien aux réalités et aux besoins locaux;

- Renforcer le niveau de qualification des maîtres;

- Aller vers une plus grande équité tant sur le plan des disparités géographiques que par rapport aux élèves aux besoins éducatifs particuliers;

- Promouvoir un projet éducatif commun à l'ensemble des écoles de Nouvelle-Calédonie."

Malgré une baisse sur les vingt dernières années, la population autochtone kanak reste majoritaire par rapport aux autres ethnies du pays. De plus, trois habitants sur quatre sont nés en Nouvelle-Calédonie, ce qui montre la forte présence des Océaniens, toutes ethnies confondues $^{8}$, dans la population calédonienne. C'est en regard de cette forte océanité de la population qu'il convient d'analyser les résultats de l'école calédonienne.

Comme le montre le graphique $\mathbf{1}$, le niveau de formation de la population calédonienne a fortement progressé en vingt ans (1989-2009). On note en particulier que la population sans diplôme a été divisée par deux.

Cependant, on retrouve là encore de fortes disparités entre les provinces. En effet, non seulement la province Sud conserve son " avance " dans l'accès au diplôme, mais l'augmentation du niveau de formation ne s'est pas non plus opérée au bénéfice des mêmes diplômes selon les provinces. On observe un déplacement vers la droite de la distribution de la population au sein des différents diplômes qui est beaucoup plus fort pour la province Sud que pour les deux autres provinces.

- En province Sud, on observe une élévation du niveau de diplôme au profit des bacheliers et les diplômés du supérieur ;

- En province Nord et province des Iles Loyauté, cela concerne surtout les diplômes professionnels inférieurs au baccalauréat.

Lélévation du niveau de diplôme est à relier à la politique de rééquilibrage qui a engendré le développement des structures et des formations. La Nouvelle-Calédonie a répondu aux besoins de scolarisation ${ }^{9}$ en construisant des établissements scolaires et en créant des postes d'enseignants. Au nom du rééquilibrage, des collèges et lycées ont été construits et/ ou rénovés dans le Nord et aux Iles Loyauté.

8. Y compris les Européens nés en Nouvelle-Calédonie.

9. Entre 1990 et 2010 , le nombre d'enfants scolarisés ( $1^{\text {er }}$ et 2 sd degré du privé et du public) a augmenté de $25 \%$. 
Graphique 1

Répartition de la population (de plus de 14 ans) par province en fonction du diplôme le plus élevé obtenu en 1989 et en 2009
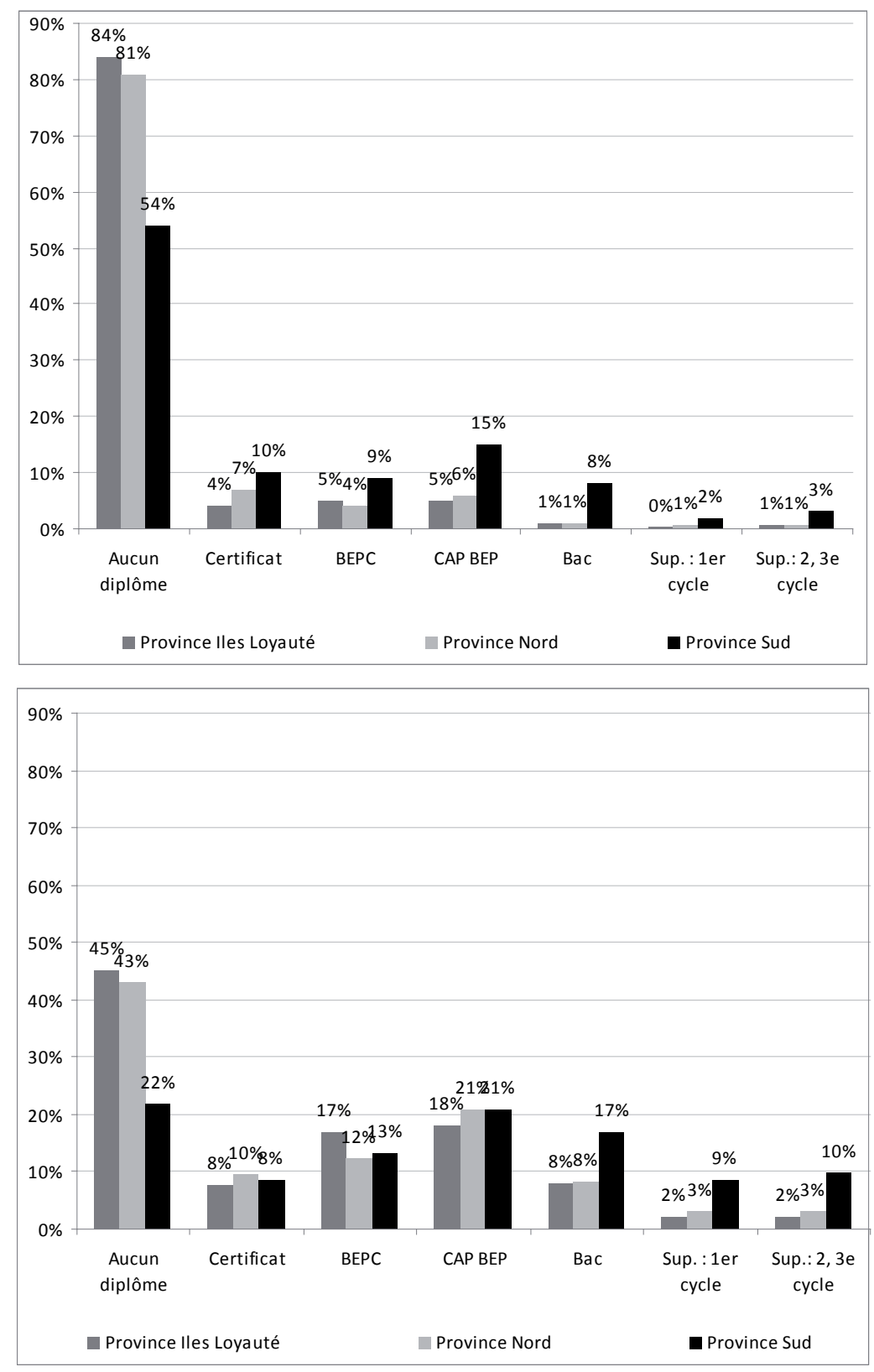

Lecture : En 1989, 84 \% des habitants de la province des lles Loyauté n'ont aucun diplôme ; ce taux est de 45 \% en 2009. Source : ISEE, Recensements de 1989 et 2009.

Sigles : BEPC : brevet des collèges ; CAP-BEP : certificat d'études professionnelles et brevet d'études professionnelles. 
Cette massification évidente de l'accès au diplôme s'est-elle accompagnée d'une démocratisation qualitative ? La question de l'égalité des chances devant l'école devient alors centrale.

En effet, ces résultats doivent être nuancés par les grandes disparités entre les communautés. Déjà, en 1985, Kohler et Wacquant (1985) soulignaient : « un enfant mélanésien ${ }^{10}$ a 2,3 fois moins de chances objectives d'obtenir un CAP qu'un enfant européen ; il a trois fois moins de chances de glaner un BEPC, six fois moins de réussir un bac technique et douze fois moins de devenir bachelier des sections classiques. "

Avant de retracer, en Nouvelle-Calédonie, l'évolution des inégalités devant l'école depuis vingt ans, la partie suivante présente le développement de la mesure des inégalités, essentiellement dans la littérature anglo-saxonne.

\section{Mesurer les inégalités ethniques devant l'école : un enjeu politique central}

\subsection{De rares études en France}

La littérature internationale (théorique et appliquée) sur le thème de la ségrégation, qu'elle soit sociale ou ethnique, est abondante. La littérature française, quant à elle, se caractérise par une quasi-absence d'études sur la mesure des inégalités ethniques et cela en raison de "l'exception française " relative aux statistiques ethniques ${ }^{11}$. Cependant, l'intérêt pour la dimension ethnique des inégalités a cru au cours de ces dernières années, notamment grâce à la mise à disposition d'ensembles de sources statistiques plus précises et plus riches (Aeberhardt, Fougère, Pouget et Rathelot, 2010). Comme le note Felouzis (2003), les discriminations liées à l'origine ethnique des individus font partie de l'expérience quotidienne des immigrés et des citoyens français issus de l'immigration. Cette limite à la mesure de la dimension ethnique conduit alors à une situation paradoxale dans laquelle la variable ethnique est devenue une catégorie à part entière de la perception de l'école et des établissements, sans pour autant être analysée en tant que telle. Cela revient aussi à occulter les phénomènes de ségrégation et de discrimination, et évite d'en décrire l'am-

10. La Mélanésie englobe la Nouvelle Calédonie.

11. L'intérêt des chercheurs français s'est alors d'avantage focalisé sur l'analyse des inégalités sociales (voir Valet, 2010, pour la présentation des principaux jalons de la recherche internationale en sociologie dans ce domaine). Alors que l'effet de l'allongement général des études est spectaculaire, la démocratisation qualitative de l'école est, elle, bien plus modeste. Certains travaux la tiennent pour réelle quoique limitée (Vallet, 1999 ; Duru-Bellat et Kieffer, 2008 ; Thélot et Vallet, 2000), alors que d'autres la jugent inexistante ou trop faible pour être significative (Goux et Maurin, 1995 ; 1997). 
pleur ainsi que les conséquences sur le parcours scolaire des élèves et plus généralement sur les mesures de réussite sociale. La mesure des discriminations est ainsi en passe de devenir l'un des enjeux politiques centraux dans les sociétés multiculturelles en ce début de XXIe siècle ; cependant, elle n'est pas exempte de débats car elle implique la délimitation des unités prises en considération, et donc la définition de l'ethnicité (voir Simon, 2008).

\subsection{Une littérature anglo-saxonne abondante}

La littérature anglo-saxonne sur le thème des inégalités ethniques est elle bien plus fournie. Nous nous focaliserons ici sur les résultats des récentes études américaines et présenterons les analyses réalisées en Australie et en Nouvelle-Zélande en raison du parallèle possible avec la situation calédonienne.

Aux États-Unis, le thème de la concentration, du regroupement des ethnies est abordé dès les années 1920 à l'Ecole de Chicago. À partir des années 1940, une nouvelle génération de travaux plus quantitatifs débouche rapidement sur une série d'indicateurs, de mesures de la ségrégation. Lélaboration de nouveaux indicateurs est toujours en développement (voir notamment Echenique et Fryer, 2007). Disposer de mesures chiffrées claires et facilement interprétables établissant le degré de ségrégation de la population noire, que ce soit sur le plan résidentiel, scolaire, ou encore sur le marché du travail, revêt une importance de premier ordre (Borjas, 1995, Fryer, 2010). Les disparités entre Noirs et Blancs sont persistantes aux USA (Heckman, 2011). Ces disparités s'opposent aux croyances américaines d'égalité, d'opportunité et de mobilité sociale (Echenique, Fryer, et Kaufman, 2006). Les études ont en effet montré que les inégalités dans les résultats scolaires apparaissent dès le très jeune âge (Fryer et Levitt, 2004) ; de même, l'environnement, dès le jardin d'enfants, a un effet significatif sur la position sur le marché du travail (Chetty, Friedman, Hilger, Saez et Schanzenbach, 2011).

En Amérique Latine, les travaux de McEwan (2004 et 2008), McEwan et Trowbridge (2006), Parker, Rubalcava et Turel (2005) et Sakellariou (2008), mettent en évidence l'importance de la langue maternelle dans l'apprentissage et dans les inégalités devant l'école.

En Australie, les travaux des chercheurs du Centre for Aboriginal Economic Policy Research (Australian National University, Canberra) mettent en évidence l'évolution de la situation des Australiens autochtones (Aborigènes et autochtones du détroit de Torrès) et allochtones (tous les autres : Européens, Asiatiques, Insulaires du Pacifique, ...) en termes de caractéristiques socio-économiques et d'accès à l'emploi (voir en particulier Altman, Biddle et Hunter, 2009 ; Biddle, Taylor et Yap, 2009). À partir des données des recensements de 1971 à 2011, ces auteurs révèlent que les inégalités ethniques se réduisent lentement, en particulier dans le domaine de l'éducation. Cette conclusion contredit l'idée de l'échec des politiques de réduction des inégalités (Pholi, Black, et Richards, 2009), mises en place depuis la fin des années 80 par le gouvernement australien. 
De la même manière, en Nouvelle-Zélande, des politiques de 'Closing the gap' (réduction des inégalités) ont été instaurées à destination de la population Maori (Johnston, Poulsen et Forrest, 2005). En utilisant les indices de dissimilarité ${ }^{12}$ de Duncan, Chapple (1999 et 2000) montre une lente mais progressive convergence des populations Maori et non-Maori en termes de dotation en éducation (réduction des disparités dans l'accès aux diplômes depuis vingt ans). "Ces mesures des inégalités intergroupes présentent un écueil : elles occultent totalement les variations intra-groupes, qui ne sont pas négligeables, en particulier au vue de la taille de certains groupes" (Chapple, 2000) (cf. note 5).

Enfin, notons que dans l'une des rares études de comparaisons internationales des inégalités ethniques, Darity et Nembhard (2000) montrent que les inégalités ethniques sont universelles; elles sont présentes et apparaissent remarquablement similaires dans une grande variété de pays (étude sur un échantillon de treize pays ${ }^{13}$ de différents niveaux de développement économique). Les auteurs précisent également que si les groupes discriminés sont souvent numériquement minoritaires, ils constituent parfois la majorité de la population.

\section{Les inégalités ethniques augmentent avec le niveau de diplôme}

Au-delà de l'approche descriptive (partie 1), mettant en évidence une amélioration générale des niveaux de formation, il convient d'apprécier quantitativement la part respective de l'allongement général des études (la massification) et de l'affaiblissement éventuel du lien entre communauté d'appartenance et diplôme (la démocratisation qualitative) dans ce phénomène. Ainsi, comme l'ont fait Selz et Vallet (2007) ou Duru-Bellat et Kieffer (2008) pour la France, il est possible d'évaluer l'évolution qualitative de la démocratisation de l'école en Nouvelle-Calédonie.

Pour mesurer l'ampleur des inégalités, nous utiliserons deux types d'indicateurs courants dans la littérature, d'une part des différences entre taux (méthode additive), d'autre part des rapports de chances (odds ratios).

12. En comparant deux distributions, l'indice de dissimilarité mesure la part des individus, dans une catégorie qui devraient la quitter pour que celle-ci ait une composition identique à celle du reste de la population. Cet indice est compris entre 0 et 1 . Il vaut zéro en situation idéale.

13. Australie, Belize, Brésil, Canada, Inde, Israël, Japon, Malaisie, Nouvelle-Zélande, Afrique du Sud, Trinidad et Tobago, et les Etats Unis. 
Tableau 1

Evolution de la répartition de la population de 14 ans et plus en fonction du diplôme le plus élevé obtenu

\begin{tabular}{|l|c|c|c|c|c|c|c|}
\hline $\begin{array}{l}\text { Communauté } \\
\text { d'appartenance }\end{array}$ & Année & $\begin{array}{c}\text { Aucun } \\
\text { diplôme }\end{array}$ & CEP & BEPC & CAP, BEP & BAC & $\begin{array}{c}\text { Diplôme } \\
\text { ens. sup. }\end{array}$ \\
\hline \multirow{3}{*}{ Kanak } & 1989 & $80 \%$ & $6 \%$ & $5 \%$ & $7 \%$ & $1 \%$ & $0,2 \%$ \\
& 1996 & $66 \%$ & $10 \%$ & $7 \%$ & $14 \%$ & $3 \%$ & $1 \%$ \\
& 2004 & $63 \%$ & n.d. & $9 \%$ & $19 \%$ & $7 \%$ & $2 \%$ \\
& 2009 & $41 \%$ & $9 \%$ & $15 \%$ & $23 \%$ & $9 \%$ & $3 \%$ \\
\hline \multirow{5}{*}{ Non Kanak } & 1989 & $49 \%$ & $10 \%$ & $10 \%$ & $16 \%$ & $9 \%$ & $6 \%$ \\
& 1996 & $31 \%$ & $12 \%$ & $9 \%$ & $21 \%$ & $13 \%$ & $14 \%$ \\
& 2004 & $36 \%$ & n.d. & $11 \%$ & $20 \%$ & $16 \%$ & $18 \%$ \\
& 2009 & $20 \%$ & $8 \%$ & $13 \%$ & $19 \%$ & $18 \%$ & $23 \%$ \\
\hline \multirow{3}{*}{ Ensemble } & 1989 & $62 \%$ & $8 \%$ & $8 \%$ & $12 \%$ & $6 \%$ & $4 \%$ \\
& 1996 & $44 \%$ & $11 \%$ & $8 \%$ & $18 \%$ & $9 \%$ & $9 \%$ \\
& 2004 & $46 \%$ & n.d. & $10 \%$ & $19 \%$ & $12 \%$ & $12 \%$ \\
\hline \multirow{3}{*}{ Différence de } & 2009 & $28 \%$ & $8 \%$ & $14 \%$ & $20 \%$ & $14 \%$ & $15 \%$ \\
pourcentages & 1989 & 31 & -4 & -5 & -9 & -8 & -6 \\
(Kanak - non Kanak) & 1996 & 35 & -3 & -2 & -7 & -10 & -13 \\
& 2004 & 27 & n.d. & -2 & -1 & -9 & -16 \\
\hline
\end{tabular}

Source : ISEE, Recensements de 1989, 1996, 2004 et 2009.

Lecture : en 1989, 80 \% des Kanaks n'ont aucun diplôme, en 2009, ils sont 41 \%.

Sigles : CEP : certificat d'études primaires ; BEPC : brevet des collèges ; CAP : certificat d'aptitude professionnelle ; BEP : brevet d'études professionnelles.

Le Tableau 1 met en évidence que :

- Pour toutes les communautés, entre 1989 et 2009, on assiste à une forte progression du niveau de diplôme obtenu ;

- Jusqu'en 2004, les Kanaks sont moins diplômés que les non-Kanaks (tous diplômes confondus) ;

- En 2009, les différences de pourcentages entre Kanak et non Kanak deviennent positives pour tous les diplômes inférieurs au baccalauréat ; cela signifie qu’à partir de cette date, les Kanaks sont, en pourcentage, plus nombreux à obtenir comme diplôme le plus élevé, des CEP, BEPC, CAP, BEP (respectivement certificat d'études primaires, brevet des collèges, certificat d'aptitude professionnelle, brevet d'études professionnelles).

- Les non-Kanaks sont, eux, toujours plus nombreux à obtenir, comme diplôme le plus élevé, le baccalauréat ou un diplôme de l'enseignement supérieur. Ces évolutions 
montrent qu'il semble y avoir un "plafond de verre " pour les Kanaks dans l'accès au baccalauréat général et plus encore à l'enseignement supérieur ;

- Lécart entre Kanak et non-Kanak, mesuré par les différences de pourcentages, s'accroît aux extrémités du spectre des qualifications (aucun diplôme d'une part et diplômes du supérieur d'autre part);

- Entre 1989 et 2009, l'écart entre les communautés reste stable pour l'obtention du baccalauréat ;

- Entre 1989 et 2009, l'écart entre les communautés se creuse pour l'obtention d'un diplôme du supérieur.

Ces résultats montrent que si le niveau général d'éducation a beaucoup évolué lors de ces vingt dernières années, l'augmentation de ce niveau a été plus rapide pour les Kanaks que pour les non-Kanaks (on lit par exemple que le pourcentage de diplômés du supérieur est passé de $6 \%$ à $23 \%$ chez les non-Kanaks et de $0,2 \%$ à $3 \%$ chez les Kanaks).

Pour savoir si l'élévation du niveau moyen de formation s'est accompagnée d'une réduction des inégalités ( rééquilibrage »), le tableau 2 présente les rapports de chances relatives entre les deux groupes pour différents niveaux de diplôme.

Tableau 2 Evolution des rapports de chances relatives Non-Kanak / Kanak dans l'obtention du
diplôme (population de 14 ans et plus)

\begin{tabular}{|l|c|c|c|}
\cline { 2 - 4 } \multicolumn{1}{c|}{} & $\mathbf{1 9 8 9}$ & \multicolumn{2}{c|}{2009} \\
\cline { 2 - 4 } \multicolumn{1}{c|}{} & Odds Ratios & Odds Ratios & $\begin{array}{c}\text { Odds Ratios } \\
\text { conditionnels }\end{array}$ \\
\hline Diplôme (tous diplômes) & 4,2 & 2,8 & 2,6 \\
\hline Baccalauréat (toutes séries) & 12,5 & 4,8 & 4,4 \\
\hline Baccalauréat général & 15,2 & 6,1 & 5,5 \\
\hline Baccalauréat technologique et professionnel & 5,4 & 1,5 & 1,9 \\
\hline Diplômes enseignement supérieur & 36,0 & 9,4 & 8,7 \\
\hline
\end{tabular}

Source : ISEE, Recensements 1989 et 2009.

Lecture : en 2009, un non-Kanak a 4,8 fois plus de chances qu'un Kanak d'être bachelier plutôt que de rester non bachelier. En contrôlant pour l'âge, le sexe et la province de résidence, ce rapport est de 4,4.

Note : en 1989, les données individuelles ne sont pas disponibles pour estimer les régressions et calculer les odds ratios conditionnels.

Ces résultats mettent en évidence de fortes inégalités ethniques dans l'accès aux diplômes. Ils confirment également une certaine démocratisation de l'accès aux diplômes : les rapports de chances relatives baissent fortement en vingt ans. Cette évolution est visible aussi sur la base des différences de pourcentages (tableau 1). 
C'est pour le baccalauréat technologique et pour les diplômes du supérieur que cette baisse est la plus forte ${ }^{14}$.

Les odds ratios varient fortement lorsque l'on distingue le type de diplôme. Les résultats montrent que les écarts augmentent avec le niveau du diplôme : écart de 1 à 2,8 pour n'importe quel diplôme et de 1 à 9,4 pour les diplômes du supérieur.

De même, lorsque l'on s'intéresse au type de baccalauréat, on observe que l'écart est fort dans le cas du baccalauréat général $(6,1)$, mais faible dans le cas du baccalauréat technologique $(1,5)$.

Lapparente réduction des inégalités est donc à relativiser lorsque l'analyse est menée pour une définition plus précise des diplômes. Ainsi, les inégalités ne disparaissent pas mais se transforment.

Les écarts de diplôme entre les deux populations, que nous venons de mettre en évidence, sont potentiellement dus à des différences de caractéristiques observables. Des régressions logistiques permettant de contrôler ces caractéristiques observables sont ensuite estimées. Le tableau 2 présente les odds ratios conditionnels aux autres facteurs pouvant expliquer l'accès au diplôme. Malheureusement, comme nous l'avons déjà souligné, les données dont nous disposons ne permettent pas de mesurer l'origine sociale des individus. Les seuls facteurs explicatifs disponibles, outre la communauté d'appartenance, sont l'âge, le sexe et la province de résidence.

Comme le montre le tableau 2, pour tous les diplômes, la prise en compte de quelques variables de contrôle réduit les rapports de chances, impliquant que la communauté d'appartenance n'est pas le seul facteur explicatif de l'obtention des diplômes ; cependant, toutes choses égales par ailleurs, la communauté d'appartenance a toujours un impact significatif sur le diplôme obtenu, qui est croissant avec le niveau de diplôme.

Dans la littérature australienne et néo-zélandaise, deux approches sont principalement développées pour tenter d'expliquer les inégalités de réussite scolaire entre autochtones et non autochtones : l'approche culturaliste (l'école et les méthodes pédagogiques occidentales ne sont pas adaptées aux autochtones) et l'approche socio-économique (l'origine sociale et l'origine géographique des autochtones differrent de celles des non-autochtones et les pénalisent). Pour la Nouvelle-Calédonie, Gorohouna (2011) montre que, sur un échantillon de 1600 jeunes (18-30 ans) de la province Nord, avant toute considération culturelle, les désavantages économiques et de capital humain des parents expliqueraient la faible réussite scolaire des Kanaks.

On observe ainsi en Nouvelle-Calédonie, comme en France, un déplacement des inégalités ; celles-ci ne se situent plus aujourd'hui dans l'accès au baccalauréat, mais se retrouvent

14. Le nombre de Kanaks diplômés du supérieur en 1989 étant très faible, toute augmentation apparait très importante (ils étaient 99, ils sont 2041 en 2009). 
dans l'hétérogénéité des types de baccalauréat ; les Kanaks sont plutôt titulaires d'un baccalauréat technologique ou professionnel, les non-Kanaks d'un baccalauréat général. En France, cela se traduit par le maintien des inégalités dans l'accès au supérieur (Duru-Bellat et Kieffer, 2008). En effet, la première vague de démocratisation s'est traduite par une concentration accrue des nouveaux bacheliers de milieu populaire dans les baccalauréats professionnels ; cela a induit une limitation des possibilités d'orientation dans l'enseignement supérieur. En particulier, l'accès aux filières d'élite n'a connu aucune démocratisation parmi les bacheliers.

Ceci limite la portée de la démocratisation de l'enseignement, puisque les débouchés ne dépendent pas du niveau de diplôme, mais du type de filière suivie.

Le processus scolaire qui aboutit à ces résultats commence dès l'école maternelle, au sein de laquelle les enfants sont accueillis comme des enfants dont les pré-requis linguistiques seraient ceux de n'importe quel autre enfant francophone. La partie suivante présente les résultats des différentes évaluations du parcours scolaire de ces jeunes.

\section{Des inégalités identifiées très tôt dans la scolarité}

En nous intéressant aux résultats présentés par les institutions scolaires, nous souhaitons montrer la vision institutionnelle de l'école calédonienne. Les données utilisées dans cette partie, issues du Vice-rectorat ou de l'UGPE (Union générale des parents d'élèves), s'appuient sur une méthode d'identification spatiale (les provinces) ou patronymique.

\subsection{Les évaluations en CE2 : faible acquisition des fondamentaux pour les Kanaks}

Le rapport présenté par la Direction de l'Enseignement de la Nouvelle-Calédonie (DENC, 2008), en août 2008, montre que les provinces Nord et Iles Loyauté obtiennent les résultats les plus faibles pour les fondamentaux, en français et en mathématiques ; ils se situent en moyenne en-dessous de $50 \%$. On constate ainsi que, dès le début de la scolarité, les enfants Kanaks n'ont pas acquis les fondamentaux nécessaires pour la poursuite d'une scolarité normale, même si la situation s'améliore avec le temps ${ }^{15}$.

15. A chaque niveau, il conviendrait d'avoir acquis, a minima, $75 \%$ des fondamentaux pour poursuivre une scolarité normale. 
Graphique 2

Evolution de l'évaluation en fin de CE2 $\left(^{*}\right)$ par province

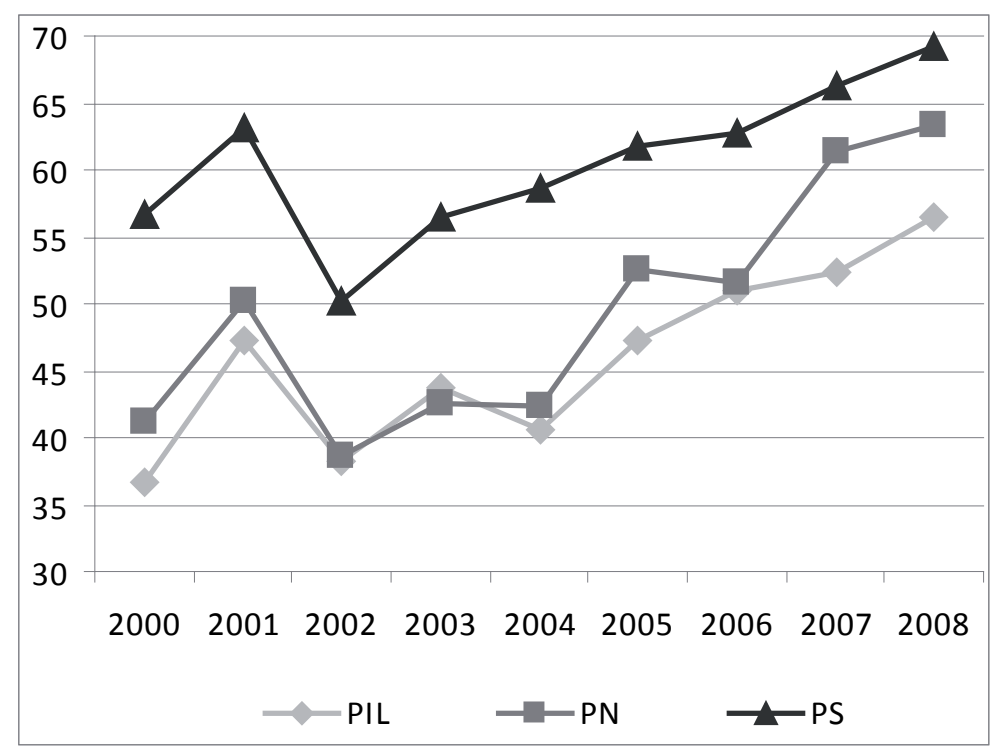

Francais

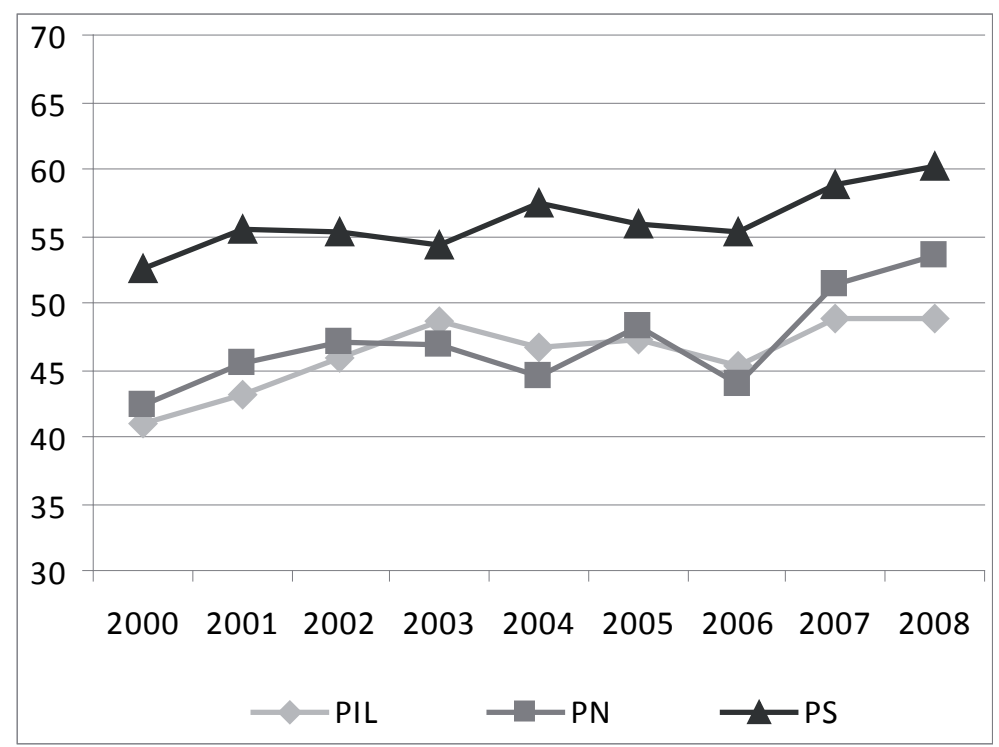

\section{Mathématiques}

Source : Direction de l'Enseignement de la Nouvelle-Calédonie (DENC, 2008).

Lecture : En 2008, en moyenne, les élèves de CE2 de la province Sud ont acquis $69 \%$ des fondamentaux en français.

Légende: $\mathrm{PS}=$ Province Sud, $\mathrm{PN}=$ Province Nord, PL = Province des Îles Loyauté. $\left.{ }^{*}\right)$ : CE2 : Cours élémentaire Zème année. 


\subsection{Les évaluations à l'entrée en $6^{\text {ème }}$ : les écarts se creusent entre provinces}

L'analyse des résultats des évaluations à l'entrée en $6^{\text {ème }}$ montre que l'écart entre les provinces se creuse. La population scolaire des provinces Nord et Iles Loyauté obtient en moyenne $36,6 \%$ de réussite en français et $32 \%$ en mathématiques ${ }^{16}$.

Lécart existant et se répétant au fil des années ne permet pas à ces jeunes d'accéder à la " réussite scolaire " développée par le système, notamment en ce qui concerne les séries générales : $E S, S$ et $\mathrm{L}$, présentées comme celles qui permettent les meilleurs choix d'orientation. Particulièrement la série $S$ (scientifique), présentée comme celle de tous les choix possibles.

\subsection{L'orientation en seconde : les Kanak sont majoritairement orientés vers les filières professionnelles}

Le document du Vice-Rectorat de Nouméa (Vice Rectorat, 2008) montre que le taux d'orientation en seconde générale et technologique est de 34,2\% pour la province nord, $42 \%$ pour la province des îles Loyauté. Le commentaire est à souligner. En effet, selon le Vice-Rectorat : " la promotion de l'enseignement professionnel, comme voie de réussite scolaire et d'insertion, qui a constitué un axe majeur de la politique éducative de ces dernières années, explique en partie ce décalage. " Une question s'impose : pourquoi, alors, cette politique éducative vers les filières professionnelles ne fonctionne-t-elle pas pour les jeunes de la province Sud, qui ont eux un taux d'orientation en seconde générale " proche de celui de la Métropole» (respectivement 58 et $60 \%$ ) ?

16. Il est admis que pour suivre une scolarité normale, il convient d'avoir acquis $75 \%$ des fondamentaux à l'entrée en $6^{\text {ème }}$. 
Graphique 3

Evolution de l'évaluation à l'entrée en $6^{\text {ème }}$ par province

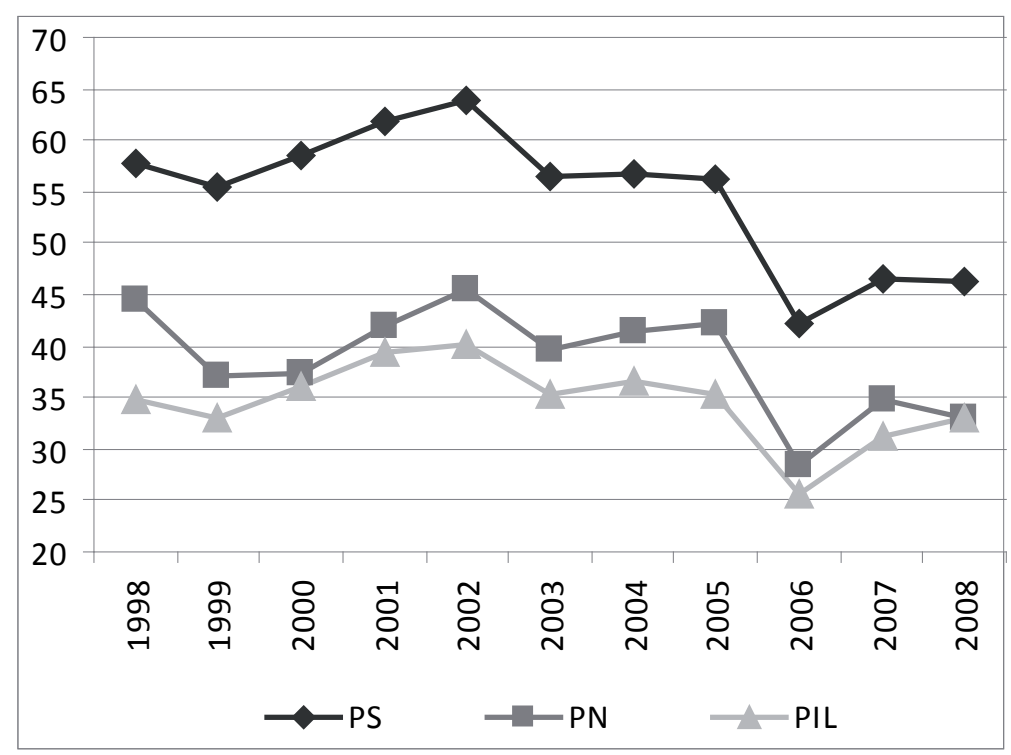

Francais

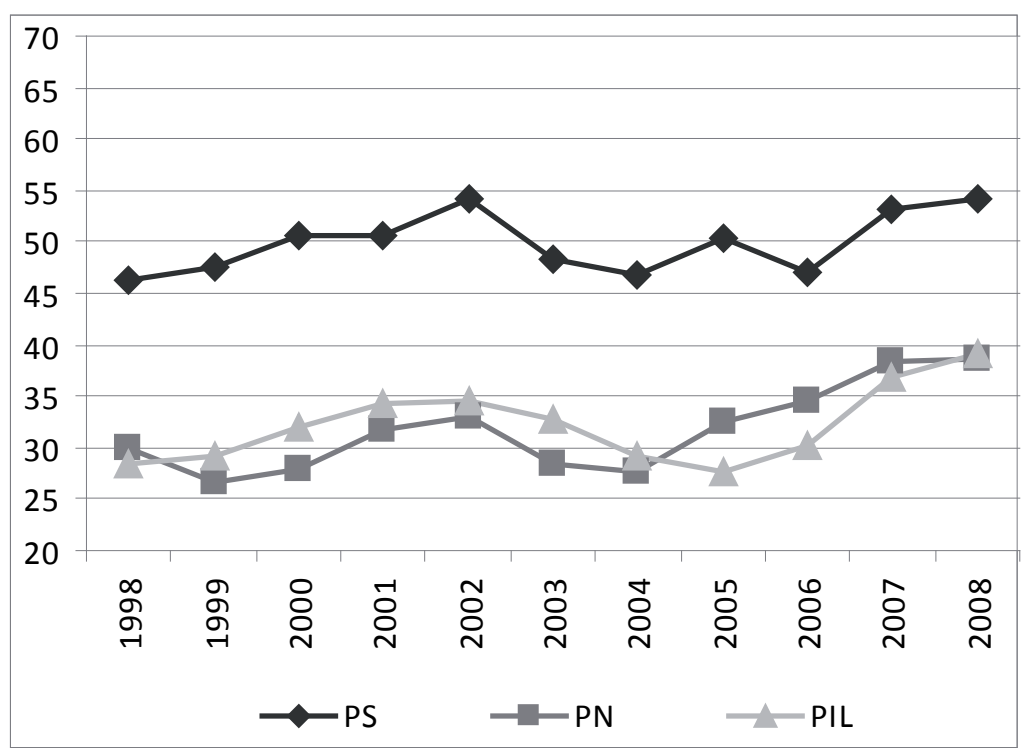

\section{Mathématiques}

Source : Vice-Rectorat, Nouméa, 2008.

Légende : PS : Province Sud, PN : Province Nord, PIL : Province des lles Loyauté.

Exemple de lecture : en 2008, en moyenne, les élèves de 6ème de la province Sud ont acquis $46 \%$ des fondamentaux en français. 
Graphique 4

Taux d'orientation vers la $2^{\text {nde }}$ générale et technologique en 2008

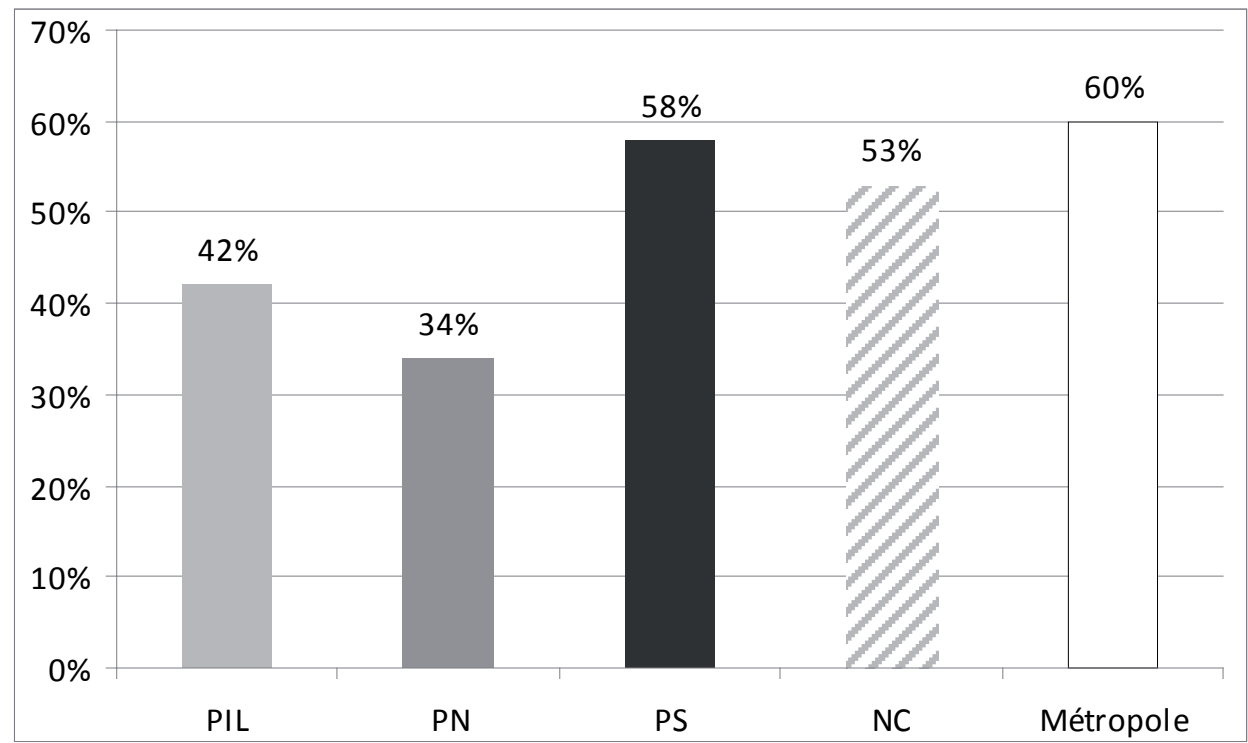

Graphique 5

Taux d'orientation vers l'enseignement prof. (BEP, CAP) en 2008

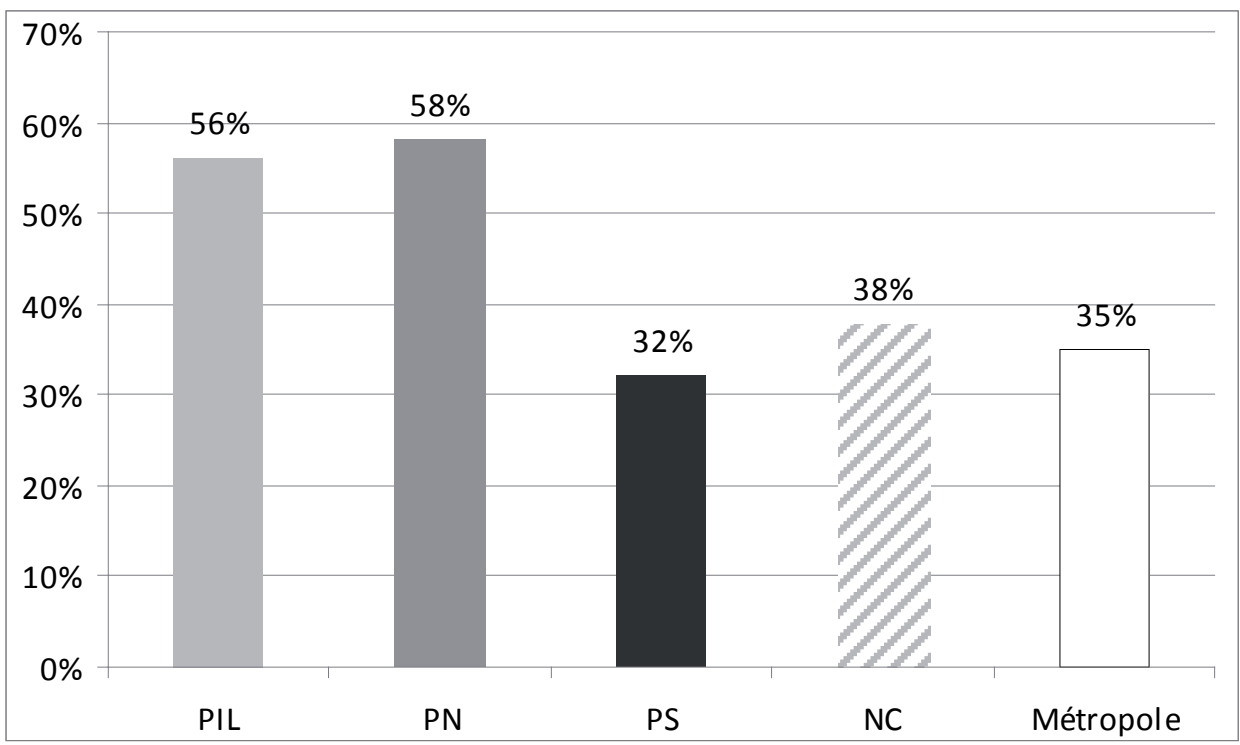

Source : Vice-Rectorat, Nouméa, 2008.

Légende : PIL : Province des Iles Loyautés ; PN : Province Nord ; PS : Province Sud ; NC : moyenne des trois provinces de la Nouvelle-Calédonie. Lecture : En 2008, en moyenne, $42 \%$ des élèves de la province Sud sont orientés vers une seconde générale et technologique.

Sigles : BEP : brevet d'études professionnelles; CAP : certificat d'études professionnelles. 


\subsection{Les séries du baccalauréat : sous-représentation des Kanaks et Océaniens ${ }^{17}$ en série $S$}

Globalement, il y a, évidemment, de plus en plus de bacheliers en Nouvelle-Calédonie. Nous avons vu, précédemment, la grande disparité de la répartition de ces bacheliers selon les communautés. Qu’en est-il pour les séries du baccalauréat?

Ne pouvant disposer officiellement de données par ethnie pour affiner la réflexion, l'UGPE (Union Générale des Parents d'Elèves) effectue, sur la base des résultats au baccalauréat présentés par le Vice-Rectorat, une analyse patronymique des bacheliers qui permet de dresser le graphique 6 pour l'année 2009. On constate d'une part, la forte présence des Européens en séries générales et particulièrement en série $S$, ainsi que la sous-représentation des Kanaks et Océaniens dans ces séries. D’autre part, la forte présence des bacheliers kanaks et océaniens en séries technologiques et professionnelles est patente.

Graphique 6

Réussite au Baccalauréat 2009

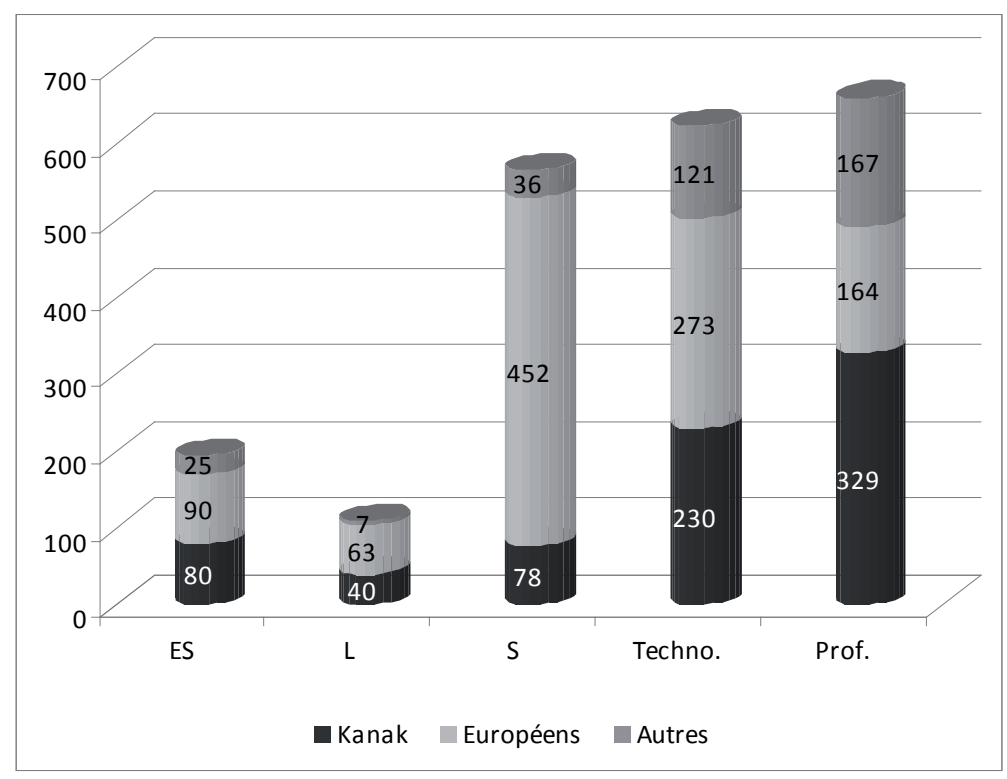

Source : Calcul UGPE.

Lecture : En 2009, 80 élèves Kanak ont obtenu un baccalauréat de série ES. La catégorie « Autres » désigne tous les élèves hors Kanaks et Européens (Walisiens, Futuniens, Polynésiens, Vietnamiens, Indonésiens, ...).

17. Les Océaniens non Kanak sont majoritairement Wallisiens, Futuriens et Polynésiens. 


\section{Conclusion}

Cette étude a permis de mettre en évidence tant la réduction des inégalités ethniques devant l'école en Nouvelle-Calédonie, depuis vingt ans, que leur persistance.

Les résultats montrent que plus on procède à une analyse désagrégée, plus les inégalités sont marquées. Le calcul de rapports de chances relatives montre que les écarts entre les communautés augmentent aux extrêmes de la distribution des diplômes (pas de diplôme d'une part et diplômes du supérieur d'autre part). Conséquence directe des inégalités dans l'acquisition des diplômes, en 2009, on observe que 66 \% des non-Kanak de 15-64 ans ont un emploi, contre $45 \%$ des Kanaks.

\section{L'échec de l'égalité des chances de l'école calédonienne: constat et tentatives d'explications}

L'école calédonienne, en calquant le modèle de l'école française, construit une inégalité de fait quant à la réussite scolaire et par conséquent la réussite sociale et professionnelle des autochtones.

On retrouve le fonctionnement développé en 1863 par le Gouverneur Guillain : l'enseignement général pour les Européens et l'enseignement professionnel pour les Kanaks et les Océaniens.

On est donc amené à conclure que, même si le système peut se réclamer d'une élévation du niveau de diplôme, donc d'une démocratisation de l'école, les résultats selon le type de diplôme acquis révèlent que les Kanaks n'ont pas les mêmes chances de réussite, d'insertion sociale et professionnelle.

Ainsi, le fonctionnement de l'école calédonienne, reproduction in extenso de celui de l'école française, implique que les jeunes Kanaks sont, dans la grande majorité, orientés vers les séries technologiques et professionnelles dès la sortie du collège.

En France, la première vague de démocratisation s'est traduite par une concentration accrue des nouveaux bacheliers de milieu populaire dans les baccalauréats professionnels ; cela a limité des possibilités d'orientation dans l'enseignement supérieur. En particulier, l'accès aux filières d'élite n’a connu aucune démocratisation parmi les bacheliers.

Cette situation n'est pas sans conséquence sur les perspectives de développement économique, alors que les théories modernes de la croissance font justement du capital humain le cœur de la croissance et du développement. La nécessité d'assurer une juste répartition des revenus de la croissance est un objectif crucial qui doit permettre de consolider une stabilité sociale, indispensable au développement équilibré et durable de la NouvelleCalédonie. Dès lors, comment atteindre cet objectif avec une école qui est encore source de fortes inégalités? 
Cette analyse présente quelques limites importantes qu'il conviendra de combler dans une prochaine étude. Tout d'abord, la non-prise en compte de l'hétérogénéité intra-groupe ; le groupe constitué des non-Kanaks est en effet très hétérogène ; il regroupe de nombreuses communautés (Européenne, Indonésienne, Ni-Vanuatu, Tahitienne, Vietnamienne, Wallisienne, Futunienne, ...) qui présentent des situations bien différentes. Ensuite, les régressions logistiques ayant servi à " corriger " les odds ratios des caractéristiques observables des individus n'incluent pas de variables de contrôle de l'origine sociale (CSP - catégorie sociale et professionnelle - ou diplôme des parents). Il convient d'affiner l'analyse pour déterminer la part respective de l'origine sociale et de l'origine ethnique dans les destinées scolaires.

\section{Bibliographie}

Aeberhardt R., Fougère D., Pouget J. et Rathelot R. (2010), «L'emploi et les salaires des enfants d'immigrés ", Economie et Statistique, n 433.

Altman J.-C., Biddle N., and Hunter B.H. (2009), "Prospects for 'Closing the Gap' In Socioeconomic Outcomes for Indigenous Australians?”, Australian Economic History Review, 49(3).

Beauchemin C., Hamel C., Lesné M. et Simon P. (2010), "Les Discriminations : une Question de Minorités Visibles ", Population et Société, 466.

Biddle N., Taylor J. and Yap M. (2009), "Are the Gaps Closing? Regional Trends and Forecasts of Indigenous Employment”, Australian Journal Of Labour Economics, 12(3).

Borjas G.J. (1995), "Ethnicity, Neighbourhoods, and Human-Capital Externalities”, The American Economic Review, 85 (3).

Broustet D., Rivoilan P. (2012), "Vingt ans de mutation de l'emploi ", ISEE, Synthèse $n^{\circ} 22$.

Chapple S. (1999), "Explaining Patterns of Disparity Between Mäori and non-Mäori Employment Chances", Labor Market Bulletin.

Chapple S. (2000), "Maori Socio-Economic Disparity”, Political Science, 52.

Chetty R., Friedman J. N., Hilger N., Saez E., Whitmore Schanzenbach D., and Yagan D. (2011), "How Does Your Kindergarten Classroom Affect Your Earnings? Evidence From Project STAR”, NBER Working Paper, n 16381.

COMEDD (2010), Inégalités et discriminations. Pour un usage critique et responsable de l'outil statistique, Rapport du Comité pour la mesure de la diversité et l'évaluation des discriminations, février.

Darity W. and Nembhard C. (2000), "Racial and Ethnic Economic Inequality: The International Record", The American Economic Review, 90(2). 
DAvid C. (2011), Les singularités humaines : les recensements pour quoi faire? Le métissage, Communication au colloque sur "Le destin des collectivités politiques d'Océanie ", Nouméa, mars, à paraître.

DENC (2008), 2000 - 2008, L'évolution de l'école primaire publique en Nouvelle-Calédonie depuis le transfert de compétences. Quelques repères pour mesurer le chemin parcouru, Rapport.

Duru-Bellat M. et Kieffer A. (2008), « Du baccalauréat à l'enseignement supérieur en France : déplacement et recomposition des inégalités ", Population, vol. 63.

Echenique F. and Fryer R. (2007), "A Measure of Segregation Based on Social Interactions”, Quarterly Journal of Economics, 122(2).

Echenique F., Fryer R. and Kaufman A. (2006), “Is School Segregation Good or Bad?”, American Economic Review, May.

Felouzis G. (2003), "La Ségrégation ethnique au collège et ses conséquences », Revue Française de Sociologie, 44(3).

FRYER R. (2010), "Racial Inequality in the 21st Century: The Declining Significance of Discrimination", Forthcoming in the Handbook of Labor Economics Volume 4.

Fryer R. and Levitt S. (2004), “The Causes and Consequences of Distinctively Black Names", Quarterly Journal of Economics, CXIX.

Gorohouna S. (2011), Dynamique des inégalités dans un pays pluriethnique. Le cas de la Nouvelle-Calédonie, thèse pour le doctorat de sciences économiques, université Paris 1 - Panthéon - Sorbonne.

Goux D. et Maurin E. (1995), «Origine sociale et destinée scolaire. L'inégalité des chances devant l'enseignement à travers les enquêtes FQP 1970, 1977, 1985 et 1993 ", Revue Française de Sociologie, 36(1).

Goux D. et Maurin E. (1997), «Démocratisation de l'école et persistance des inégalités ", Economie et Statistique, 306 (Juin).

Guillain C. (1863), "Arrêté n 125 du 15 octobre 1863 ", Bulletin Officiel de NouvelleCalédonie, Bibliothèque Nationale de Paris. pp. 234 - 238.

HADJ L. (2010), «En 2008, le niveau de vie des plus riches est 7,9 fois supérieur au niveau de vie des plus modestes ", ISEE, Synthèse budget consommation des ménages 2008, $\mathrm{n}^{\circ} 18$.

Heckman J.J. (2011), “The American Family in Black and White: A Post-Racial Strategy for Improving Skills to Promote Equality”, NBER Working Paper, n 16841.

Johnston R., Poulsen M. and Forrest J. (2005), “The Residential Segregation of New Zealand Maori in Comparative Perspective: An 'Ecology of Social Inequality'?", New Zealand Population Review, 31(2). 
Kohler J.-M., Wacquant L. (1985), L'école inégale. Éléments pour une sociologie de l'école en Nouvelle-Calédonie, Institut Culturel Mélanésien, Nouméa, coll. "Sillon d'igname ", pp. 36-37.

McEwan P. (2004), "The Indigenous Test Score Gap in Bolivia and Chile", Economic Development and Cultural Change, 53(1).

McEwan P. (2008), "Can Schools Reduce the Indigenous Test Score Gap? Evidence from Chile", Journal of Development Studies, 44 (10).

McEwan P. and Trowbridge M. (2006), "The achievement of indigenous students in Guatemalan primary schools", International Journal of Educational Development, 27 (1).

Parker S., Rubalcava L. and Turel G. (2005), "Schooling Inequality and Language Barriers", Economic Development and Cultural Change, 54(1).

Pholi K., Black D. and Richards C. (2009), "Is 'Close the Gap' a useful approach to improving the health and wellbeing of Indigenous Australians?", Australian Review of Public Affairs, 9(2).

Sakellariou C. (2008), "Peer effects and indigenous/non-indigenous early test-score gap in Peru", Education Economics, 16 (4).

Salaün M. (2005), L'école indigène. Nouvelle-Calédonie, 1885-1945, Presses universitaires de Rennes, 280 p.

Simon P. (2008), " La mesure des discriminations ethniques et raciales : comparaisons internationales ", in Fassin E. et Halpérin J.-L (dir.) Discriminations : pratiques, savoirs et politiques, La Documentation française, Paris.

Selz M., Vallet L.-A. (2007), «Évolution historique de l'inégalité des chances devant l'Ecole : des méthodes et des résultats revisités ", Education \& Formations, 74.

ThÉLot C. et Vallet L.-A. (2000), « La Réduction des inégalités sociales devant l'Ecole depuis le début du siècle ", Economie et Statistique, 334(4).

VALLET L.-A. (1999), "Quarante années de mobilité sociale en France. L'évolution de la fluidité sociale à la lumière de modèles récents ", Revue Française de Sociologie, 40(1).

VALlet L.-A. (2010), "Expansion des systèmes éducatifs et dynamique des inégalités sociales devant l'enseignement : Quelques jalons de la recherche comparative en sociologie », Economie et Statistique, 433-434.

Vice-Rectorat de Nouvelle-Calédonie (2008), Eléments pour un diagnostic du système éducatif en Nouvelle-Calédonie, Nouméa. 